\title{
Sinus Cavernous Syndrome as A Presentation of Nasopharyngeal Cancer - A Case Report
}

\section{Popovska $\mathrm{H}^{\mathbf{1}}$, Petrov $\mathrm{I}^{\mathbf{2}}$ and Kiteva-Trencevska $\mathrm{G}^{\mathbf{2}}$}

${ }^{1}$ University Clinic of Neurology, Skopje, Macedonia

${ }^{2}$ Professor, University Clinic of Neurology, Skopje, Macedonia

*Corresponding Author: Popovska H, University Clinic of Neurology, Skopje, Macedonia.

DOI: $10.31080 /$ ASMS.2020.04.0567
Received: January 20, 2020

Published: February 14, 2020

(C) All rights are reserved by Popovska H., et

al.

\begin{abstract}
Nasopharyngeal cancer (NPC) is relatively rare cancer in the western world, comprising 0,2 - 0,5\% of all cancers and $2 \%$ of all head and neck squamous cell carcinomas. Also, it is rarely implicated as a cause of cranial nerve involvement. Because of its place of origin, the nasopharynx, which is very difficult to examine, because of unspecified clinical manifestation in the early stages of the disease and because the postnasal space is frequently occupied by normal lympho-epithelium, which make the differentiation even more difficult, this type of carcinoma remain undiagnosed until the late stages of the disease. Computed tomography (CT) scan and magnetic resonance imaging (MRI) are the investigation of choice for accurate tumor mapping and detection of possible tumor extension. We report one case with a nasopharyngeal carcinoma, presenting with a syndrome of cavernous sinus, diagnosed by neurologist in the late stadium of the disease, even though there was a control examination by otorhinolaryngologist before establishing the accurate diagnosis.
\end{abstract}

Keywords: Sinus Cavernous Syndrome; Nasopharyngeal Carcinoma; Delayed Diagnosis; Magnetic Resonance Imaging (MRI)

\section{Case Report}

We present a case of a 69 year-old man who was admitted to our hospital, because of a recent neurological deficit, recognized by his general practitioner two weeks ago. It was manifested as an acute -onset headache, associated with a pain in the right eye, drooping of the right eyelid, inward deviation of the eye, as well as binocular horizontal diplopia. Also, he complained about gradually progressive dysphagia, nasal speech and tiredness during walking, over the past two years. In the meanwhile, when there was no obvious neurological deficit, the patient consulted a specialist in otorhinolaryngology, and a diagnosis of rhinitis was given. A few medications were prescribed.

From his medical history it is worth to mention that two years ago he underwent surgical treatment of nasal polyps, without any complications after the surgery. The other medical history was no significant. There was a history of nicotin dependece in the past forty years, with an abstinence period of two years, after the surgical treatment of nasal polyps.

After physical examination, we suspected on syndrome of cavernous sinus in addition to bulbar syndrome. So the possible etiology that we were thinking of was for sure malignancy (much more of secondary process, than primary) and, as a second option, inflammatory /infectious disease (sarcoidosis, tuberculosis, Wegener granulomatosis, fungal or other chronic infection), taking in consideration the age of the patient.

The laboratory tests for complete blood cell count, blood glucose, liver and renal function tests, erythrocyte sedimentation rate and C-reactive protein were normal. Cerebrospinal fluid parameters were normal. CT scan of the brain showed mild bilateral tem- 
poroparietal cortical atrophy, without any finding that could explain the symptoms. And, MRI of the brain revealed a tumor mass with origin from nasopharynx, involving the sphenoid sinus as well as ethmoid sinus. The tumor was making erosion to the clivus and sella turcica, without clear evidence of intracranial protrusion. There was infiltration to both cavernous sinuses, surrounding the left internal carotid artery approximately 180 degree of the vessel circumference, without luminal compromise. The part of the tumor located in the posterior part of ethmoid sinus was making significant compression to the left optical nerve. There were chronic inflammatory changes of both mastoids. There were no enlarged cervical lymph nodes (Figure 1 and 2).

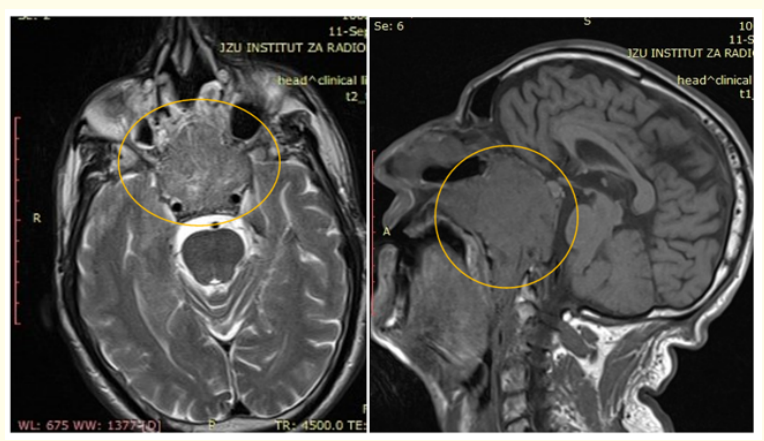

Figure 1 and 2

After consultation with an otorhinolaryngologist, fiberoptic endoscopy and multiple biopsies were performed, we had the histopathologic diagnosis- squamous cell nasopharyngeal cancer, keratinizing type. The patient underwent radiotherapy and chemotherapy was planned. The prognosis was not optimistic.

\section{Discussion}

Nasopharyngeal carcinoma (NFC) is a rare no lymphomatous squamous cell carcinoma(SCC), that occurs in the epithelial lining of the nasopharynx, with three histopathologic subtypes, proposed by the World Health Organization (WHO): keratinizing SCC (type I), non-keratinizing (type 2) and undifferentiated carcinoma, the most common type (type 3) [1].

NFC is a rare malignancy in most parts of the world. It accounts for $2 \%$ of all head and neck squamous cell carcinomas. The incidence of NFC, generally, outside of endemic areas of Southeast Asia, is less than $1 / 100000$ people, predominantly in the male population. In Southeast Asia, the NFC is the third most common malignancy among men, with an incidence of 50 per 100 000. Emigration from high- to low-incidence areas reduces the incidence of NFC in first generation Chinese, but it still remains at seven times the rate in Caucasians [2]. These epidemiological facts represent how the interactions between underlying genetic susceptibility and surrounding environmental factors play a critical role in the etiopathogenesis of the NFC. It is currently believed that EpsteinBarr virus (EBV) plays a critical role in transforming nasopharyngeal epithelial cells into invasive tumors [1].

The nasopharynx lies behind the nasal cavities and above the soft palate. On each lateral wall of the nasopharynx is the pharyngeal opening of the pharyngo-tympanic tube. Behind and above the posterior margin of the tube, between it and the posterior pharyngeal wall, lies the lateral pharyngeal recess (fossa of Rosenmuller). This is the most complex of all walls because of its close association with the content of the parapharyngeal space and the lymphatics. The nasopharynx is a clinical blind spot in the middle of the skull base. It is a difficult area to see, being situated in a relatively big and inert space where only air and mucus are in transit [4-7].

Most common place of origin of NFC is the nasopharyngeal recess (fossa of Rosen muller). Then it can spread submucosally with early infiltration of the deep cervical spaces. Local growth of the tumor can involve the Eustachian tube orifice or m. levator veli palatini that commonly results with serious otitis media. Beyond the nasopharynx, NPC spreads along well-defined routes: anteriorly (invading the nasal cavity and pterygopalatine fossa through the sphenopalatine foramen), laterally into the parapharyngeal space (involving the masticator muscles will cause trismus or infiltration of mandibular nerve will result with denervation atrophy of the muscles of mastication), posteriorly infiltrating the retropharyngeal And prevertebral spaces (involving the nasopharyngeal carotid space and compromising the IX, X, XI and XII nervs), superiorly (via the foramen lacerum and foramen ovale or through direct skull base erosion into the intracranial cavity) and inferiorly (spreading along the submucosal plane or through the retropharyngeal space or extending into the oropharynx) [1,3].

Perineural spread of head and neck tumors is a well described phenomenon in the surgical and radiological literature. It refers to extension of benign or malignant tumor along a nerve, which may reach a significant distance from the primary lesion. Perineural 
spread carries a grave prognosis. Up to $30-45 \%$ of patients with extensive perineural spread may remain asymptomatic, with normal nerve function at clinical examination [8].

Cervical nodal metastasis is very common in NPC, and often the initial presenting complaint. From distant metastasis (up to 41\%), common sites include bone (20\%), lung (13\%), and liver (9\%) [1].

Cranial nerve involvement can be caused by direct extension of the NPT invading the skull base or intracranial infiltration via the foramina at the base of the skull. Intracranial infiltration occurs primarily through the foramen lacerum or foramen ovale [9].

Perineural spread through the foramen ovale is an important route, which explains why there is an evidence of sinus cavernous involvement, without evidence of skull base erosion (in this case the CT scan was normal, which means the bone integrity of cranium is intact) [10].

All these data confirm and support the reason why early detection of NFC is difficult, even though early diagnosis is very important to achieve optimum results of treatment. Some studies state that there are two factors as the cause of misdiagnosis of NPC, which are: patient factors (patient delay) and health system factors (professional delay). Improper diagnosis, lack of suspicion of NPC by professional health practitioners, especially in low rate incidence area, play a very important role in late diagnosis [11].

Therefore, medical practitioners must improve their diagnostic capabilities especially in differential diagnosis and to consider the possibility of NPC. It is suggested that increased knowledge and understanding of the disease among neurosurgeons, neurologists and ENT surgeons, would improve the prognosis of patients with NPC. Patients and their families should also be aware of these complaints, especially in regions with high incidence of NPC. The hospital administration should have a system of tracking down high risk patients who default $[9,11,12]$.

\section{Conclusion}

In our case, the CT scan was with a relatively normal finding. On MRI we reveal a bizarre tumor mass invading the nasopharynx, nasal choanae, both cavernous sinuses, sphenoid sinus, ethmoid sinus. Taking in consideration the extension of the tumor, we can agree that the clinical manifestation is really poor (the patient had symptoms from local extension and unilateral syndrome of cavern- ous sinus). So, we must point out that NPC has unique patterns of spreading, with features that keep the tumor unrevealed for a long time. Taking in consideration and being aware of the above mentioned factors that lead to misdiagnosis/delayed diagnosis, and how to overcome them, we can contribute to earlier detection of the disease and improve the prognosis of patients with NPC.

\section{Conflict of Interest}

The authors have no conflict of interests in this article.

\section{Bibliography}

1. Cheng K Ong and Vincent FH Chong. "Neoplasms of the Nasopharynx". Head and Neck Cancer Imaging, edited by Robert Hermans, 2 nd ed., Springer (2012): 163-180.

2. Keiji Tabuchi., et al. "Early Detection of Nasopharyngeal Carcinoma”. International Journal of Otolaringology (2011).

3. JJ Lu., et al. "Natural History, Presenting Symptoms, and Diagnosis of Nasopharyngeal Carcinoma Nasopharyngeal cancer". Multidisciplinary menagement, edited by L.W. Brady, H.-P. Heilmann, M. Molls, C. Nieder, Springer (2010): 41-51.

4. Ahmed M Domaa and Hanan A Gad. "The Clinical Manifestations Of Nasopharyngeal Cancer In Libya-A Comparative Study". Middle East Journal of Applied Sciences 1.1 (2011): 1-4.

5. Beasley P. "Anatomy of the pharynx and esophagus". In: Michael G, editor. Scott-Brown's OtoLaryngology. 6th edition, vol.6. Oxford: Butterworth-Heinemann (1997): 1-40.

6. Chuan TC. "Nasopharynx (The postnasal space)". In: Hibbert J, editor. Scott-Brown's OtoLaryngolog. 6 th ed, vol.5. Oxford: Butterworth-Heinemann (1997): 1-30.

7. Chong VFH.) Neoplasms of the nasopharynx". In: Hermans R (ed) Head and neck cancer imaging. Springer, Berlin Heidelberg New York (2006):129-142.

8. CK Ong and VFH Chong. "Imaging of perineural spread in head and neck tumors". Cancer Imaging 10 (2010): S92-S98.

9. Mehmet Turgut., et al. "Importance of cranial nerve involvement in nasopharyngeal carcinoma. Aclinical study comprising 124 cases with special reference to clinical presentation and prognosis". Neurosurgical Review21 (1998): 243-248.

10. Agrawal A., et al. "Nasopharyngeal Carcinoma Presenting with Cavernous Sinus Involvement, Trigeminal Neuralgia and Middle Ear Effusion". International Journal of Clinical Pharmacy 2.4 (2009): 203-207. 
11. Marlinda Adham., et al. "Delayed diagnosis of nasopharyngeal carcinoma in a patient with early signs of unilateral ear disorder". Medical Journal of Indonesia 23.1 (2014): 52-57.

12. Jern-Lin Leong., et al. "Factors contributing to delayed diagnosis in nasopharyngeal carcinoma”. The Journal of Laryngology and Otology 113.7 (1999): 633-636.

\section{Assets from publication with us}

- Prompt Acknowledgement after receiving the article

- Thorough Double blinded peer review

- Rapid Publication

- Issue of Publication Certificate

- High visibility of your Published work

Website: https://www.actascientific.com/

Submit Article: https://www.actascientific.com/submission.php

Email us: editor@actascientific.com

Contact us: +919182824667 